\title{
Distribución anatómica de ramas subendocárdicas en mamífero no humano, primer reporte
}

\begin{abstract}
D Rafael Coello Cuntó ${ }^{1}$; Liz Carranza Zambrano ${ }^{2}$; Oscar González Arreaga ${ }^{2}$; María Auxiliadora Marmolejo Araujo ${ }^{2}$; Andrea Rodríguez Salazar; Jostin Badillo Torres²; Maytte Moreira Vinueza²; Katherine Plaza Alvarado²; Jessenia López Holguín²; Juan Velásquez Apolinario²; Daniel López Gomez ${ }^{2}$; Adrián Valverde Montes de $\mathrm{Oca}^{2}$ \& Moisés Pinela Chávez².
\end{abstract}

\section{RESUMEN}

Establecer la organización de las fibras subendocárdicas es útil para determinar la conducción y propagación normal o anormal del impulso cardiaco y tiene trascendencia en el campo educativo, hemodinámico y cardiológico; su identificación ha sido posible habiéndose encontrado patrones similares en muchas especies mamíferas. Históricamente denominadas fibras de Purkinje, las ramas subendocárdicas (A.12.1.06.008) de Terminologia Anatomica son inidentificadas macroscópicamente y han sido evidenciadas con varias técnicas con distinto valor y limitaciones. Como componente de la formación académica, en el laboratorio de Anatomía Humana de la Universidad de Guayaquil se ha replicado su identificación utilizando tinta china en corazones adultos de vacunos y porcinos frescos mostrándose macroscópica e histológicamente su presencia. El nodo sinoatrial y atrioventricular son uniformes en su constitución, las fibras subendocárdicas muestran una estructura reticular con múltiples interconexiones, esto ha permitido realizar una descripción aproximada. Sus detalles de distribución y organización son importantes para interpretar los problemas de conducción como las arritmias.

Palabras clave: fascículo atrioventricular; nodo atrioventricular; ramas subendocárdicas; Taller de Preparaciones Anatómicas.

\section{Anatomical subendocardial fibers distribution in mammals, first report}

\begin{abstract}
Establishing the organization of the subendocardial fibers is useful to determine the normal or abnormal conduction and propagation of the cardiac impulse and has importance in the educational, hemodynamic and cardiological fields; their identification has been possible, having found similar patterns in many mammalian species. Historically called Purkinje fibers, the subendocardial branches (A.12.1.06.008) of Anatomical Terminology are macroscopically unidentified and have been evidenced with various techniques with different value and limitations. As a component of academic training, in the Human Anatomy laboratory of the University of Guayaquil its identification has been replicated using Chinese ink in adult hearts of fresh cattle and pigs, showing its presence macroscopically and histologically. The sinoatrial and atrioventricular node are uniform in their constitution, the subendocardial fibers show a reticular structure with multiple interconnections, this has allowed an approximate description. Their distribution and organization details are important in interpreting conduction problems such as arrhythmias.
\end{abstract}

Key words: atrioventricular bundle; atrioventricular node; subendocardial branches; Anatomical Preparations Workshop.

\section{Introducción}

Jan Evangelista Purkinje describió en 1839 y ratificó en 1845 un conjunto reticular de fibras grises en el subendocardio de una oveja que lo relacionó con el aparato motor del corazón; en 1906 se demostró que las fibras encontradas por Purkinje viajan en el interior de trabéculas musculares como un sistema de conducción que se demostró en humanos, caninos, vacunos y ovinos; por tal razón se acepta denominarlos miocitos especializados(1) (2). La distribución, estructura histológica e irrigación del sistema de conducción involucra a todas las ciencias implicadas y relacionadas con la función cardiaca con

1. Universidad de Guayaquil, Ecuador.

2. Taller de Preparaciones Anatómicas, Universidad de Guayaquil, Ecuador.

Citar como : Coello Cuntó R, Carranza Zambrano L, González Arreaga O, Marmolejo Araujo MA, Rodríguez Salazar A, Badillo Torres J, Moreira Vinueza M y col . Distribución anatómica de ramas subendocárdicas en mamífero no humano, primer reporte. Rev Peruana de Morfologia. 2020; 1(2):28-32. doi: https//doi.org/1051343/revperuanamorfologia.v1i2.273

Recibido: 2020-06-12; Aceptado: 2021-02-07

Autor corresponsal: Rafael Coello Cuntó, Phd., Mg.Esp.; Email: msdracocu@hotmail.com 
pocas variaciones en las distintas especies de mamíferos bien diferenciados (3). La anatomía del sistema de conducción cardiaca es similar en los mamíferos aunque se conocen tres tipos de organización según la especie (4). Macroscópicamente, el NAV tiene una estructura reticular y es inconfundiblemente mas pálido que el tejido vecino; el FAV tiene una longitud aproximada de $20 \mathrm{~mm}$ y calibre de $4 \mathrm{~mm}$ (5) y se inicia una vez que el NAV atraviesa el núcleo fibroso central que tiene forma redondeada, elíptica o triangular con una longitud de $2,3 \mathrm{~mm}$ y espesor de $1,1 \mathrm{~mm}$ aproximadamente; la rama derecha se presenta como una delgada prolongación del FAV (5) las fibras derechas son francamente subendocárdicas en su inicio luego se confunden con la porción muscular y finalmente se hacen superficiales cerca del fascículo arqueado; la rama izquierda tiene disposición laminar (2) y se comporta distribuyéndose en dos grupos: hacia anterior y posterior hasta las proximidades de los músculos papilares y son más largas en los jóvenes(6)(7). Disecciones han demostrado tres disposiciones del FAV: a lo largo del borde inferior de la porción membranosa del septo interventricular, dentro de la porción muscular del septo y sobre la porción membranosa. Para identificarlas se han utilizados modelos animales mamíferos diversos y tinta china(8). Para determinar la organización y distribución anatómica de las RSE se utilizaron corazones vacunos y porcinos adultos frescos intentando proponer una descripción utilizando una técnica básica que se comprobó por histología.

\section{Material y metodo}

En el Taller de Preparaciones Anatómicas (TPA), laboratorio de la Facultad de Ciencias Médicas de la Universidad de Guayaquil, área dedicada a la conservación de tejidos biológicos se procesan corazones frescos de mamíferos. Por carecer de experiencias previas se remitió el protocolo a la bibliografía existente y al resultado obtenido de las experiencias fallidas que se efectuaron en 7 muestras de vacuno y 7 de porcinos adultos tomadas de depostadero. El procedimiento incluyó : a) Limpieza con barrido mecánico de agua para movilizar restos y contenido tanto de cámaras cardiacas como de vasos intramurales; el lavado permite apreciar el aclaramiento del órgano; b) Congelamiento de la muestra por $15 \mathrm{~h}$, paso necesario para facilitar la aplicación del medio colorante; c) Descongelamiento de la muestra por 4 horas; d) Sección de los atrios, orejuelas y los troncos de la aorta y pulmonar con liberación de tejido adiposo vecino; e) Apertura de ventrículos con bisturí: ventrículo izquierdo por posterior siguiendo un plano imaginario entre los dos músculos papilares desde la base hasta $2 \mathrm{~cm}$ aproximadamente cerca el ápex; algunas cuerdas tendinosas son retiradas en caso dificulten o sean comprometidas con el corte y se mantienen las de mayor calibre, la incisión se prolonga hacia proximal siguiendo la silueta de la aorta entre las válvulas semilunar posterior e izquierda; ventrículo derecho se realiza la incisión por posterior cerca al septo y próximo a la válvula septal, luego se prolonga hacia el tronco pulmonar entre las válvulas semilunares derecha e izquierda; f) Aplicación por inyección con dispositivo de $1 \mathrm{ml}$ utilizando tinta china Pelikan $\AA$ : $5 \mathrm{ml}$ diluidos en $25 \mathrm{ml}$ de cloruro de sodio en vaso libre de turbulencias y desechable; de preferencia la aplicación deberá hacerse hacia proximal por la facilidad de identificar las fibras por transparencia o en algún caso a través de las cuerdas tendinosas, el progreso de la solución se ayuda con ligeros masajes para garantizar la aplicación tangencial de la tinta y el corazón cortado bien sujetado; todos los pasos deben ser realizados con delicadeza y con asistencia, en caso se necesite realizar nuevas punturas no dudar en hacerlo.

Las muestras fueronidentificadas macroscópicamente documentadas con videos, fotografías digitales (adjuntas en figs.1-5) posteriormente se analizaron por histología convencional y corroboradas por expertos; a) Fijación con formalina al $0,25 \%$ a $4^{\circ}$ por $48 \mathrm{~h}$; b) Deshidratación en alcohol etílico en concentraciones ascendentes; c) Diafanización con xilol; d) Inclusión en bloque de parafina y corte; e) Tinción con hematoxilina y eosina ; f) Montaje para el análisis bajo microscopia (9).

\section{Resultados}

Luego de atravesar el anillo fibroso derecho, las fibras del FAV teñidas de menor intensidad y de menor diámetro que las fibras de la pared, se organizan en formanodosa enlapartealtade los ventrículos(Figs. 1,2 y 3) y se distribuyen más ampliamente por todas las paredes subendocárdicas mostrándose muchos enlaces con el músculo cardiaco con variaciones de ordenamiento según las dos especies estudiadas sin mostrar un patrón definido; fueron observadas macroscópicamente e histológicamente establecidas como miofibrillas de menor tamaño que las vecinas, color pálido y rodeadas de un conectivo bien definido variándose su apreciación dependiendo del corte efectuado, el tinte sirvió de guía de identificación. Macroscópicamente se pudo determinar que la rama derecha, a veces más visible, se comporta como 
una cuerda de mm por el subendocardio del septum interventricular hasta llegar a la base del músculo papilar septal, posteriormente penetra en el espesor de la trabécula septomarginal y luego se arboriza finamente desde la pared septal a la pared anterior. Otras fibras se dirigen detrás del complejo valvular y hacia anterior ocupando la trabécula septomarginal; a pesar de ser visibles son más difíciles de inyectar por formar precozmente muchas figuras poligonales y francamente reticulares, su distribución es más densa hacia la salida del orificio del tronco pulmonar (Fig.4).

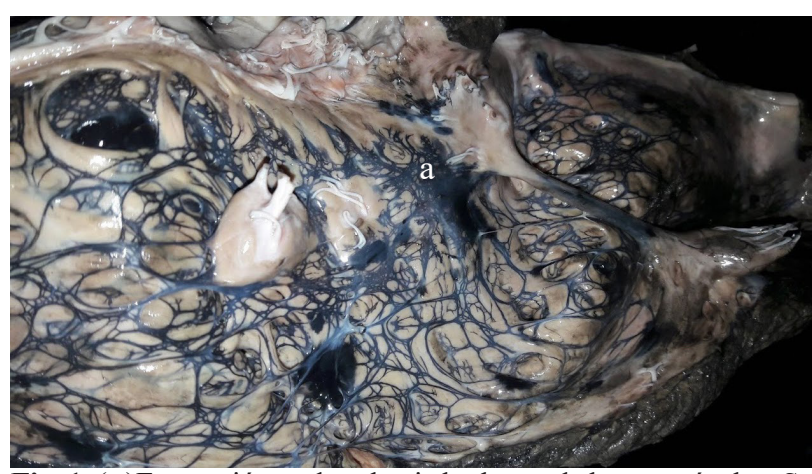

Fig. 1: (a )Formación nodosa hacia las bases de los ventrículos. Se identifica el fascículo atrioventricular en el septo interventricular.

La rama izquierda presenta divisiones muy precozmente en la parte alta de la porción muscular del septo cercanas al orificio aórtico entre la válvula anterior derecha y posterior, antes de llegar al ápex se divide en ramas anterior y posterior que se dirigen a las columnas carnosas respectivas y en dirección de las cuerdas tendinosas, también se aprecia una división ocupando cuerdas tendinosas hacia el septo. Se aprecian en el ventrículo izquierdo fibras delgadas dispuestas en forma de telaraña mientras en el ventrículo derecho son retículas alargadas que conforme se dirigen hacia la periferia se hacen más delgadas. En otra muestra se pudo observar la parte medial del ventrículo donde las fibras tienen forma circular, en ocasiones se hacen triangulares y de menor calibre, más poligonales en los pilares y más abundantes que en la base; en el septo y hacia lateral donde parecen bifurcarse formando una arborización; hacia el ápex la red es más densa y las fibras parecen confluir en forma de retícula (Fig.4).

En todas las muestras histológicas se pudo apreciar tejido conectivo rodeando las fibras teñidas y separadas del tejido muscular vecino, encontrándose algunas fibras discontinuas especialmente hacia el musculo papilar derecho.

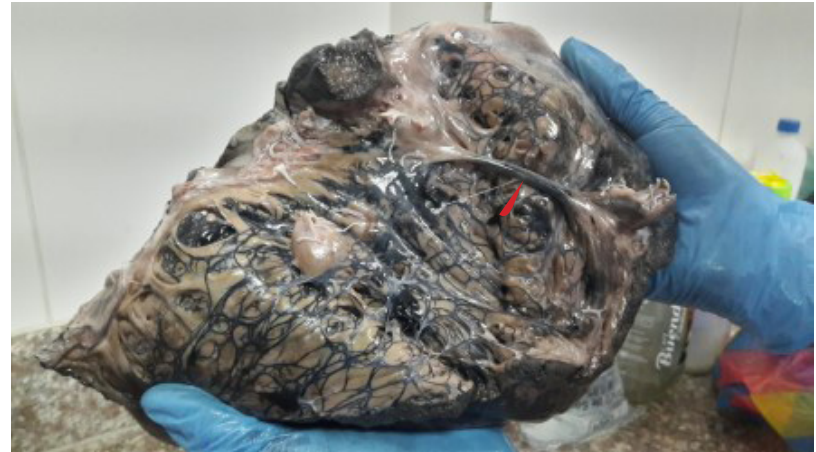

Fig. 2: Distribución reticular en ambos ventrículos. Se destaca con flecha la trabécula septomarginal.

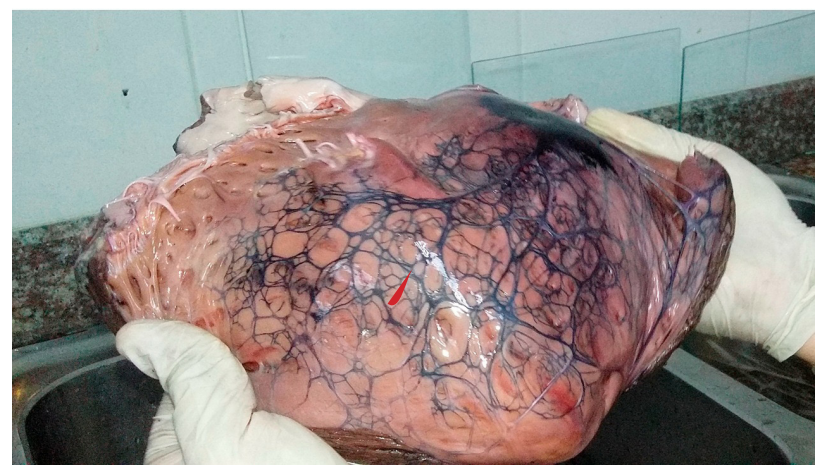

Fig. 3: Distribución plexiforme en ventrículo izquierdo. Se destaca con flecha la distribución en pared diafragmática.

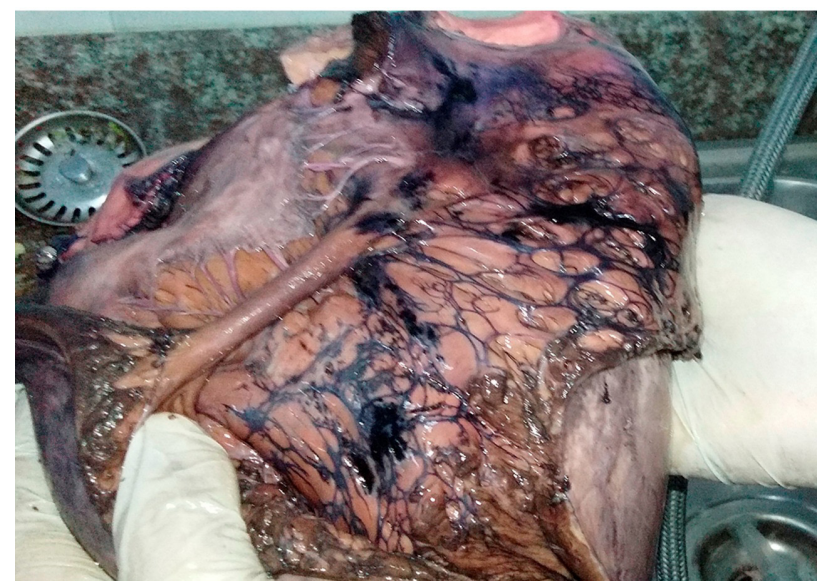

Fig. 4: Organización en ventrículo derecho. Se señala la válvula septal del complejo valvular atrioventricular derecho, cuyo territorio cercano ya no recibe fibras subendocárdicas 


\section{Discución}

Se estableció la organización reticular de las RSE (1); por su parte se identificaron fibras subendocárdicas usando tinta negra hindú(7)(13)(10), de composición similar a la actual tinta china su distribución es esencial para interpretar arritmias y alteraciones en la conducción (11) y son marcadamente diferentes en relación con la masa muscular de ambos ventrículos (12). La organización de las RSE es indiscutiblemente reticular (10)(13); últimamente se ha indicado que las fibras en el ventrículo derecho se aproximan a la base de la columna carnosa y se dirigen hacia el vértice (14). Especialmente tupidas hacia la cresta supraventricular pero más distalmente se confunden con las fibras musculares vecinas siendo más complicado hacia la izquierda por su adherencia al endocardio donde es factible identificarlas con una meticulosa disección (6). En 1959(15) se identificó las fibras de la rama izquierda del FAV con una solución yodada de lugol en humano, ratificando su estudio con histología usando tinción de carmín para destacar el gran contenido de glicógeno de las fibras; se ha encontrado comunicación entre las fibras ventriculares a través del septo (11). Se conocen tres tipos de patrones de fibras subendocárdicas según la especie: grupo I: ungulados; grupo II: humanos, caninos, gatos y monos; grupo III: roedores (4). En la rama izquierda se ha establecido una organización bifascicular para unos y otros han identificado una organización trifascicular organizados en dos territorios: anterosuperior y posteroinferior (5) hallazgos que concuerdan con los realizados por disección (3). La identificación por electromiografía e inmunohistoquímica ha establecido dos patrones de distribución de las fibras del FAV; a) En embudo continuándose como un árbol y; b) Modelo en forma de hojas acopladas a zonas discretas del ventrículo (16); en corazones de ovinos la rama izquierda se divide en dos territorios, muy parecido a los vacunos (11) muchas conexiones intramiocárdicas han sido encontradas en bovinos, aves y becerros que no existen en los humanos. También se ha encontrado fibras discontinuas entre las más superficiales y profundas en ovejas (13). En el ventrículo derecho, las fibras son identificadas agrupadas y organizadas desde la pared anterior del ventrículo hasta cerca de la válvula atrioventricular anterior (13). Obviamente la cresta supraventricular es la última porción en teñirse, lo que puede relacionarse con la función contráctil (11).

\section{Conclusion}

La identificación de las RSE es el paso inicial para determinar patrones de conducción y eventuales alteraciones así como establecer sus relaciones patológicas; la característica reticular permite identificarlas una vez se inyecte una fibra evidente a simple vista; lo recomendable es inyectarlas desde el ápex de los ventrículos por estar más aglutinadas y ser más numerosas evidenciándose fibras bien diferenciadas, algunas intermedias en diferenciación y otras de caracteres intermedios demostrando su amplia interconexión intramural en ciertas secciones, a veces una cuerda tendinosa es útil. La distribución es marcadamente reticular con aproximaciones y direcciones periféricas de distintas figuras evidenciándose la diferencia entre las dos especies estudiadas pero siempre tomando dirección de los músculos papilares formando muchas figuras poligonales; sin embargo no se aprecia una distribución constante que posiblemente con métodos de identificación complementarios se podría lograr una mejor delimitación y descripción más aún por tratarse de fibras de comportamiento tridimensional.

\section{Conflicto de interes}

los autores declaran no tener ningun conflito de interes

\section{Financiamiento}

Autofinanciado por los autores.

\section{REFERENCIAS}

1. Tawara, S., SHIMADA, M. \& SUMA, K. (. The conduction system of the mammalian heart: an anatomico-histological study of the atrioventricular bundle and the Purkinje fibers. Imperial College Press,. 2000.

2. Murillo, M., Cabrera, J. A., Pizzaro, G. \& Sánchez-Quintana, D. Anatomía del tejido especializado de conducción cardíaco. Su interés en la cardiología intervencionista. RIA, 1(2), 229-245. 2011.

3. Truex, R. C., \& Smythe, M. Q. Comparative morphology of the cardiac conduction tissue in animals. Annals of the New York Academy of Sciences, 127(1), 19-33. 1965.

4. Ono, N., Yamaguchi, T., Ishikawa, H., Arakawa, M., Takahashi, N., Saikawa, T., \& Shimada, T. Morphological varieties of the
Purkinje fiber network in mammalian hearts, as revealed by light and electron microscopy. Archives of histology and cytology, 72(3), 139-149. 2009.

5. Waller, B. F., Gering, L. E., Branyas, N. A. \& Slack, J. D. (). Anatomy, histology, and pathology of the cardiac conduction system: Part II. Clinical cardiology, 16(4), 347-352. 1993

6. Widran, J.\& Lev, M. The dissection of the atrioventricular node bundle and bundle branches in the human heart. Circulation, 4(6), 863-867. 1951.

7. Ansari, A., Yen Ho, S., \& Anderson, R. H. Distribution of the Purkinje fibres in the sheep heart. The Anatomical Record: An Official Publication of the American
Association of Anatomists, 254(1), 92-97. 1999.

8. De Almeida, M. C., Lopes, F., Fontes, P., Barra, F., Guimaraes, R. \& Vilhena, V.. Ungulates heart model: a study of the Purkinje network using India ink injection, transparent specimens and computer tomography. Anatomical science international, 90(4), 240-250. 2015

9. Montalvo, C.E. .Técnica histológica; http://bct.facmed.unam.mx/wp-content/ uploads/2018/08/3_tecnica_histologica.pdf. 2010.

10. Abramson, D. I., \& Margolin, S. . A Purkinje conduction network in the myocardium of the mammalian ventricles. Journal of anatomy, 70(Pt 2), 250. 1936. 
11. De Almeida, M. C., Araujo, M., Duque, M. \& Vilhena, V.. Crista supraventricularis Purkinje network and its relation to intraseptal Purkinje network. The Anatomical Record, 300(10), 1793-1801. 2017.

12. Sedmera, D., \& Gourdie, R. G. Why do we have Purkinje fibers deep in our heart? Physiological research, 63. 2014.

13. Cardwell, J. C., \& Abramson, D. I. The atrioventricular conduction system of the beef heart. American Journal of Anatomy, 49(2), 167-192. 1931

14. Hondeghem, L. M. \& Stroobandt, R. Purkinje fibers of sheep papillary muscle: occurrence of discontinuous fibers. American Journal of Anatomy, 141(2), 251-261. 1974.

15. Uhley, H. N. \& Rivkin, L. M. Visualization of the left branch of the human atrioventricula bundle. Circulation, 20(3), 419-421. 1959.
16. Oosthoek, P. W., Viragh, S., Lamers, W. H. \& Moorman, A. F. Immunohistochemical delineation of the conduction system. II: The atrioventricular node and Purkinje fibers. Circulation research, 73(3), 482-491. 1993. 